\title{
Modern routes for materials rapid processing
}

\author{
C.Plapcianu $^{1}$, M.C.Bartha ${ }^{1,2}$, M.Burdusel ${ }^{1,3}$ and R.Vidu $^{4 *}$ \\ ${ }^{1}$ NIMP, Magurele, 77125, Romania. \\ ${ }^{2}$ West University of Timisoara, Bd.V. Parvan 4, 300223, Timisoara, Romania \\ ${ }^{3}$ University Politehnica of Bucharest, Splaiul Independentei No 313, \\ RO060042 \\ ${ }^{4}$ UCDavis, Davis, CA 95621, USA. \\ *Corresponding author: r.vidu@ucdavis.edu
}

\begin{abstract}
Unconventional processing routes-microwave (MW) and/or spark plasma sintering (SPS)-applied for doped $\mathrm{MgB}_{2}$ and $\mathrm{Sr}_{2} \mathrm{FeMoO}_{6}$ (SFMO) type compounds, induce peculiar effects on structural and magnetic properties. $\mathrm{MgB}_{2}$ with $\mathrm{C} 60$ substitutes for $\mathrm{B}$ in the MgB2 lattice during MW synthesis and SPS sintering are possible, while SFMO samples show fine grain size and good magnetic properties. Both methods provide fast processing conditions and therefore considerable low energetic consumption
\end{abstract}

\section{Introduction}

The manufacture of advanced materials such as oxide and ceramic composites, as well as non oxide materials with improved properties by new modern routes as microwave sintering (MW) and/or spark plasma sintering (SPS) techniques, have certain advantages as follows:

- Both are fast, clean and energy efficient, and generally speeds up the synthesis and sintering processes (short dwell and processing time within a few minutes)

- MW processing originates from the interaction of the electromagnetic field with induced or permanent molecular dipoles and/or ions; the adsorbed energy is distributed all over the material, while energy loss occurs only at the surface (sample is hotter inside, colder at the surface)

- SPS technique implies pulse DC current passing through the graphite die as well as the sample, which facilitates high heating and/or cooling rate (up to 1000 $\mathrm{K} / \mathrm{min}$ )

-Both MW and SPS are sintering methods with low energetic consumption which allows a good ratio quality/price of the products manufactured by applying these techniques.

\section{General}

Improving the grain size and mechanical and thermal properties as well impose the use of new reliable technologies capable to protect the environment and save energy at the same time. Therefore our work has focused on adapting new techniques to $\mathrm{MgB}_{2}$ and $\mathrm{Sr}_{2} \mathrm{FeMoO}_{6}$ compounds synthesis and sintering.

Magnesium diboride $\left(\mathrm{MgB}_{2}\right)$ [1] has several advantages over other HTS (high temperature superconductors) type superconductors as it is composed of two elements arranged into a layered hexagonal structure, has low anisotropy, is non-toxic, inexpensive, and has a relatively high $T_{\mathrm{c}}$ of $39 \mathrm{~K}$.

$\mathrm{MgB}_{2}$ has been manufactured by various synthesis routes but the most promising results were achieved by the SPS processing [1-3]. In the case of $\mathrm{MgB}_{2}$, rapid synthesis is a useful mechanism because leads to an increased number of grain boundaries through the suppression of grain growth. A higher density of grain boundaries is well known to positively contribute to the 
enhancement of pinning and, hence, to increase $J_{c}$ in $\mathrm{MgB}_{2}$ superconductor.

The $\mathrm{Sr}_{2} \mathrm{FeMoO}_{6}$ (SFMO) double perovskite is very attractive due to the appreciable low-field magnetoresistance in granular form and a relatively high Curie temperature (410-450 K) [4-8]. Taking into account very weak values of magnetoresistance in single crystals, the role of grain boundaries has been extensively studied [9-13]. The intergrain magnetoresistance of polycrystalline $\mathrm{Sr}_{2} \mathrm{FeMoO}_{6}$ is known to depend on its grain size and the amount of insulating nonmagnetic $\mathrm{SrMoO}_{4}$ impurity formed during fabrication [14].

One of the main problems during synthesis of SFMO is the excessive reduction of $\mathrm{Fe}^{3+}$ producing $\mathrm{Fe}^{2+}$ species and even metallic iron, which can increase saturation magnetization [15]. Another problem is the phase stability at ambient conditions. In general, the method and synthesis conditions must be chosen carefully for preparing pure double perovskite with small antisite defects concentration during the reducing step as evidenced by deviation from the theoretical saturation magnetization at low temperature (4.0 $\mu \mathrm{B} /$ f.u.).

\section{$\mathrm{MgB}_{2}$ processing by $\mathrm{MW}$ and SPS unconventional routes}

$\mathrm{MgB}_{2}$ doped with fullerene (C60) was performed in a single-mode cavity microwave (MW) furnace, meaning only one mode of microwave propagation is permitted and hence the field pattern is well defined so the material can be positioned accordingly. It is worth mentioning that previous work reported in literature on conventional powder-in-tube processing was not successful in carbon chemical doping of $\mathrm{MgB}_{2}$ when using $\mathrm{C}_{60}$ [16] while unconventional Spark Plasma Sintering (SPS) produced excellent carbon substituted samples [17]. Expectations are that the influence of the MW electromagnetic field may generate positive chemical doping effects. Table 1 presents some data of the pellets obtained by MW (B5 and B6) compared with other (B7) prepared by SPS route. As shown in Table 1, lower density (73 and 85\%) is obtained for the MW samples than for the SPS $(>90 \%)$. We note that data presented previously in literature on MW synthesized samples were likely obtained in most cases by using adapted commercial multimodal microwave furnaces [17-20], while we used a single mode furnace built in the lab. MW processing allows the formation of the MgB2 phase (PDF 01-072-7019) as the main phase (Figure 1). Impurities are present, and their amount is similar or smaller than that previously reported [17] on MW synthesis. The main impurityphase is $\mathrm{MgO}$ (PDF 01-1235). Another impurity phase with the main peak located at $2 \theta=$ $44^{\circ}$ (Figure 1) was assigned to the presence of the (MgB19.615)0.971 phase (PDF 01070-8720). Because the effects of carbon substitution in $\mathrm{MgB} 2$ are missing when using C60 when conventional technologies are performed [20], it is obvious that the use of unconventional methods promotes the substitution.

Table $1 \mathrm{MgB}_{2}$ doped with fullerene processed

\begin{tabular}{|c|c|c|}
\hline $\begin{array}{l}\text { Starting materials } \\
\text { and synthesis route }\end{array}$ & $\mathrm{T}_{\text {sint. }}[\mathrm{K}]$ & $\begin{array}{c}\text { Density } \\
{\left[\mathrm{kg} \cdot \mathrm{m}^{-3}\right] /[\%]}\end{array}$ \\
\hline $\begin{array}{c}\mathrm{Mg}+2 \mathrm{~B} \text { (elements) - } \\
\mathrm{MW} \text {; B5 }\end{array}$ & \multirow[b]{2}{*}{1223} & $2190 / 83.2$ \\
\hline $\begin{array}{c}\mathrm{Mg}+1.95 \mathrm{~B}+0.05 \mathrm{C} \\
\text { (elements }+\mathrm{C}_{60^{-}} \\
\text {fullerene) }-\mathrm{MW} ; \mathrm{B} 6 \\
\end{array}$ & & $1930 / 73.3$ \\
\hline $\begin{array}{c}\mathrm{MgB}_{2}+0.05 \mathrm{C} \\
(\text { commercial } \\
\text { powder+C } \mathrm{C}_{60^{-}} \\
\text {fullerene) -SPS; B7 }\end{array}$ & 1423 & $2450 / 93.1$ \\
\hline
\end{tabular}

by SPS and/or MW routes

\section{SFMO obtained by conventional and SPS route}

The double perovskite $\mathrm{Sr}_{2} \mathrm{FeMoO}_{6}$ (SFMO) was prepared by two ways consisting of conventional solid-state reaction and SPS method. The powders from mixed oxides precursors were processed by the classic 
powder metallurgy route starting from the oxides mixture followed by sintering in conventional furnace tube and the unconventional Spark Plasma Sintering (SPS) route.

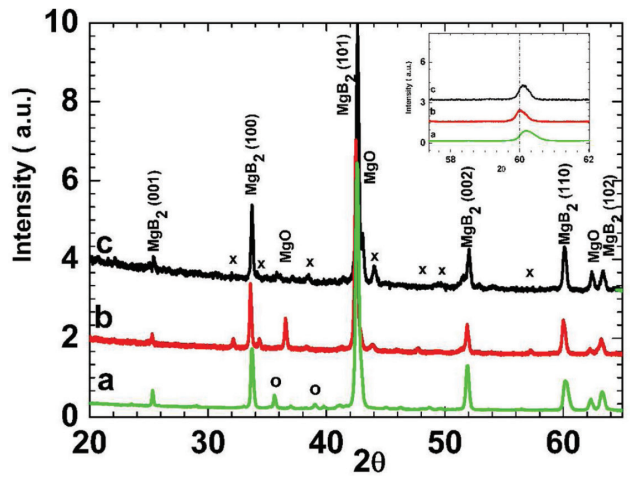

Figure 1. X-ray diffraction patterns for $\mathrm{MgB}_{2}$ samples: (a) B7 $\left(\mathrm{MgB}_{1.95} \mathrm{C}_{0.05}\right)$, SPS-doped with $\mathrm{C} 60$; (b) $\mathrm{B} 5\left(\mathrm{MgB}_{2}\right), \mathrm{MW}$; and (c) $\mathrm{B} 6$ $\mathrm{MgB}_{1.95} \mathrm{C}_{0.05}$ ), $\mathrm{MW}$-doped with $\mathrm{C} 60$. The inset shows the (110) peak of $\mathrm{MgB}_{2}$ : samples to which $\mathrm{C} 60$ had been added have the typical shift when $C$ substitutes for $B$ in the crystal lattice of $\mathrm{MgB}_{2}$. The notation of impurity phases is as follows: $\mathrm{x},\left(\mathrm{MgB}_{19.615}\right)_{0.971}$; and $\mathrm{o}, \mathrm{MgB}_{4}$.

This is likely due to the specific processes activated by the field (electrical or MW) in the unconventional methods

The structural and microstructural properties of the double perovskite SFMO powders as-prepared and ceramics were compared showing microstructures consisting of very fine grains $(0.5-0.8 \mu \mathrm{m})$ and a crystalline perovskite structure. Tetragonal SFMO pellets were prepared from the two powders by classical sintering at $1200^{\circ} \mathrm{C}, 2 \mathrm{~h}$ in $5 \% \mathrm{H} 2 / \mathrm{Ar}$ and also, by SPS at $1100^{\circ} \mathrm{C}$ followed by annealing at $1200^{\circ} \mathrm{C}$, $2 \mathrm{~h}$ in $5 \% \mathrm{H} 2 / \mathrm{Ar}$.

Figure 2 shows the XRD diagrams of the sintered pellets, named as follows: sample A1-pellet prepared from gel powder and classic sintered at $1200{ }^{\circ} \mathrm{C}, 2 \mathrm{~h}$ in $5 \% \mathrm{H} 2 / \mathrm{Ar}$; sample B1-pellet prepared from powder synthesized by conventional method and classic sintered at $1200^{\circ} \mathrm{C}, 2 \mathrm{~h}$ in $5 \% \mathrm{H} 2 / \mathrm{Ar}$; sample A2-pellet prepared from gel powder and sintered by SPS at $1100^{\circ} \mathrm{C}, 6 \mathrm{~min}$ at 50 $\mathrm{MPa}$ and then annealed at $1200^{\circ} \mathrm{C}, 2 \mathrm{~h}$ in $5 \% \mathrm{H}_{2} / \mathrm{Ar}$ and, B2-pellet prepared from powder synthesized by conventional method, sintered by SPS at $1100^{\circ} \mathrm{C}, 6 \mathrm{~min}$ at $50 \mathrm{MPa}$ and then annealed at $1200^{\circ} \mathrm{C}, 2 \mathrm{~h}$ in $5 \% \mathrm{H}_{2} / \mathrm{Ar}$. The XRD analyses revealed the formation of tetragonal $\mathrm{Sr}_{2} \mathrm{FeMoO}_{6}$ (space group I4/mmm) [21] in all samples.

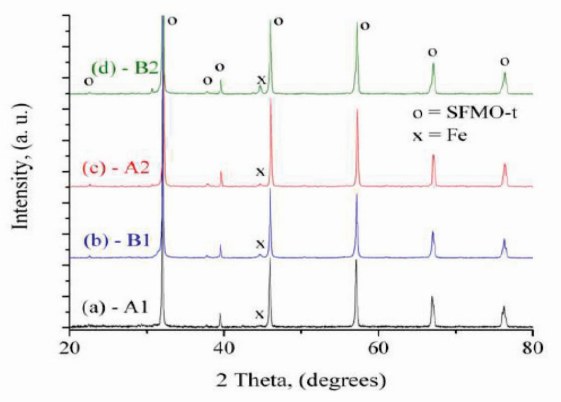

Figure 2. XRD patterns of SFMO pellets

Small quantities of impurity phases were also detected, among which Fe is the most prominent. In sample $\mathrm{A} 1$, the amount of Fe is close to the detection limit of the measurement. The XRD data were processed by Rietveld refinement using TOPAS. The main goal was to assess the iron concentration in each sample in order to correlate with the magnetic properties of the material. The resulted values of reciprocal Fe-Mo substitution in the sites were used in the theoretical estimation of magnetization.

\section{Properties}

The structure analysis of both type compounds processed by new routes revealed peculiar properties by comparison with those obtained by conventional methods. The main results are presented below

\section{1 $\mathrm{MgB}_{2}$}

Scanning electron microscopy shows that the SPS sample (Figure 3a) has grains that are smaller than those of the C60-doped 
MW sample (Figure $3 \mathrm{~b}-\mathrm{d}$ ). We could not observe

significant differences between the morphology of the doped and undoped MW samples. However, MW samples have a quite specific microstructure. Figure $3 b$ shows a microstructure composed of regions of dense structure and regions of low density with open porosity. A closer look at a higher magnification indicates the presence of compact sintered blocks (Figure 3c, inset) that resemble the morphology of SPS samples (Figure 3a), and a porous dendrite matrix partially sintered. Dendrites are composed of sintered equiaxial particles $\left(2.5-4.5 \times 10^{-7}\right.$ $\mathrm{m})$, often with round edges. Some dendrites have a one-dimensional-like shape of a needle with a length of up to $5 \times 10^{-6} \mathrm{~m}$ (Figure 3d). One end, i.e., the base that is in contact with the sample, has a larger diameter than the tip. To the best of our knowledge, such one-dimensional (1D) morphology was not observed in samples obtained by other methods, including SPS (Figure 3a). It is possible that the atypical heating in the MW process, from inside out, generates the right thermal gradient conditions for the formation of a 1D needle. In other words, MW heating through the induced thermal gradient with a low temperature at the tip of the needle promotes 1D dendrite formation. A higher temperature at the base also explains the larger diameter at the base of the needle compared to the tip[23-24].

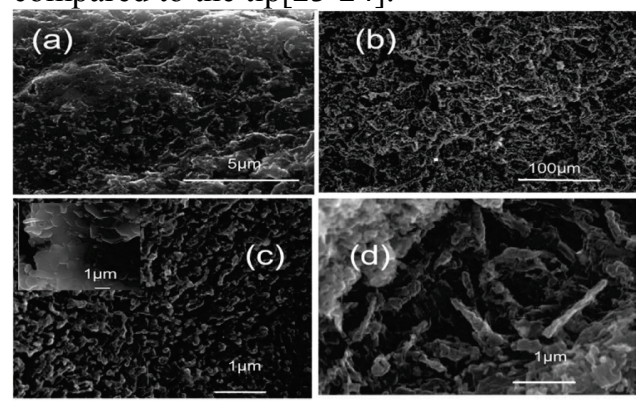

Figure 3. SEM images for (a) the $\mathrm{B} 7$ reference sample $\left(\mathrm{MgB}_{1.95} \mathrm{C}_{0.05}\right)$ obtained by SPS and (b- d) the $\mathrm{B} 6$ sample $\left(\mathrm{MgB}_{1.95} \mathrm{C}_{0.05}\right)$ obtained by MW.

\subsection{SFMO}

Compared with the morphology of $\mathrm{Sr}_{2} \mathrm{FeMoO}_{6}$ pellets obtained by classical sintering, the pellets sintered by SPS presents a more homogeneous morphology, a smaller grain size and a higher densification degree (figs. 4 and 5). The pellets sintered by spark plasma sintering shown density $>97 \%$ ot, while the pellets sintered by the conventional technique $94 \%$ pt.

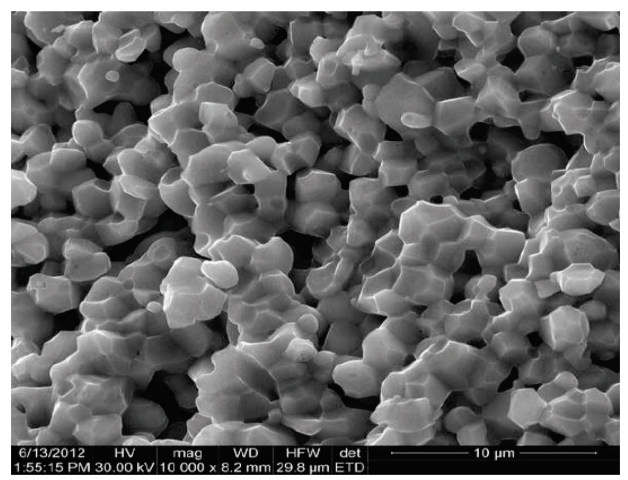

Figure 4. SEM micrographs for SFMO pellets A2 sintered by SPS at $1100^{\circ} \mathrm{C}$

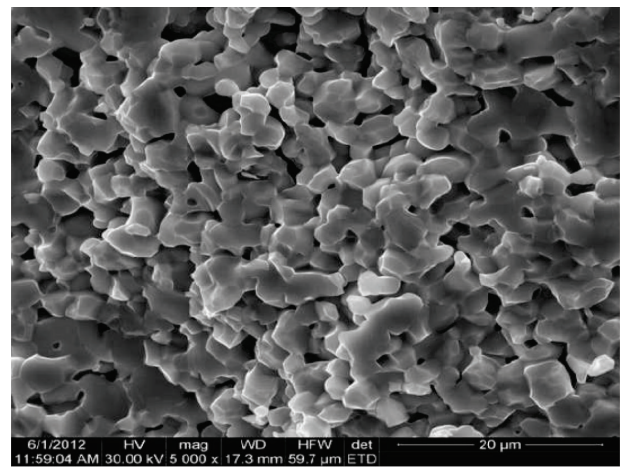

Figure 5. SEM micrographs for SFMO pellets B2 sintered by SPS at $1100^{\circ} \mathrm{C}$

The pellets presented different magnetic characteristics. The saturation magnetization of the samples prepared by sol-gel is higher than those prepared by conventional synthesis method, for the same method of sintering and, the pellets sintered by conventional method shown higher 
saturation magnetization values than the ones sintered SPS.

It has been demonstrated that the magnetization of SFMO depends strongly on the order parameter of the structure, which is influenced by the thermal processing conditions (temperature, time and atmosphere for heat treatment) [24]. Assuming one unpaired electron contributes approximately $1 \mu \mathrm{B} /$ f.u., ordered SFMO samples should have a saturated magnetic moment of $4 \mu \mathrm{B} / \mathrm{f}$.u. This value is derived from the high spin $\mathrm{Fe}^{3+}$ ion $(\mathrm{S}=5 / 2)$ coupled antiferromagnetically with the minority spin of the $\mathrm{Mo}^{5+}(\mathrm{S}=1 / 2)$ [25].

The obtained values of the total magnetic moment, listed in Table 2, correspond to the saturation magnetization of the samples. These values are lower than the theoretical one $(4 \mu \mathrm{B})$ and this fact is due to the $\mathrm{Fe} / \mathrm{Mo}$ antisite disorder existing in the double perovskitic structure. Iron location on both crystallographic positions leads to the formation of metal clusters, generating a distribution of ferrimagnetic couplings.

Table.2 Total magnetic moment calculated for SFMO sintered samples measured at $5 \mathrm{~K}$ in a $5 \mathrm{~T}$ applied magnetic field

\begin{tabular}{|l|l|l|}
\hline Sample. & $\mathrm{M}_{\text {sat }}(\mathrm{emu} / \mathrm{g})$ & $\mu_{\mathrm{exp}} / \mathrm{f} . \mathrm{u}$ \\
\hline A2 & 35.47 & 2.70 \\
\hline B2 & 35.09 & 2.69 \\
\hline
\end{tabular}

\section{Conclusions}

MgB2 bulk samples undoped and doped with fullerene were synthesised using a single-mode laboratory microwave furnace. The microstructure of MW samples is less uniform than that of the SPS samples. MW samples are composed of dense sintered blocks along with a unique dendrite matrix. Some dendrites have 1D acicular shape with a large diameter at the base and are formed likely because of the particular MW heating conditions, i.e., heating developed from the inside out. Structural and superconducting properties strongly support the idea that
C60 substitutes for $\mathrm{B}$ in the $\mathrm{MgB} 2$ lattice during

MW synthesis. This result is similar to that of the field-activated method, SPS, but differs from that of conventional synthesis for which $\mathrm{C}$ substitution did not occur when $\mathrm{C} 60$ is added to $\mathrm{MgB} 2$. We believe that field-activated processes promote substitution.

Tetragonal SFMO pellets were prepared from mixed oxide powders and sol-gel precursors by classical sintering at $1200^{\circ} \mathrm{C}$, $2 \mathrm{~h}$ in $5 \% \mathrm{H}_{2} / \mathrm{Ar}$ and, by SPS at $1100^{\circ} \mathrm{C}$ followed by annealing at $1200{ }^{\circ} \mathrm{C}, 2 \mathrm{~h}$ in $5 \% \mathrm{H}_{2} / \mathrm{Ar}$. The SPS sintered pellets showed higher density than the pellets sintered by the conventional technique. X-ray diffraction and transmission electron microscopy have evidenced the formation of tetragonal SFMO as major phase and small amounts of $\mathrm{Fe}$ segregated which influences the magnetic properties of the samples prepared by various processes. The results of magnetic measurements indicated that the saturation magnetization of the samples prepared by sol-gel is higher than those prepared by conventional synthesis method, for the same method of sintering. Also, the pellets sintered by conventional method shown higher saturation magnetization values than those sintered by spark plasma sintering.

\section{Acknowledgment}

The authors would like to thank the Sectorial Operational Program Human Resource Development under the project POSDRU/159/1.5/S/137750 and POSDRU/159/1.5/S/132395: "Doctoral and postdoctoral programs - support for the research competitiveness in sciences" for supporting the work dedicated to SFMO type compounds.

\section{References}

1) J.Nagamatsu, N. Nakagawa, T. Muranaka, Y. Zenitani and J. Akimitsu, Nature 410 (6824). (2001). 63. 
2) C. Dong, J. Guo, G. C. Fu, L. H.Yang and H. Chen, Supercond. Sci. Technol. 17 (12). (2004), L55

3) Xia, Q.; Yi, J.; Peng, Y.; Luo, S.; Li, L. Microwave direct synthesis of MgB2 superconductor. Mater. Lett. 2008, 62 (24), 4006-4008.

4) K.L. Kobayashi, T. Kimura, H. Sawada, K. Terakura, Y. Tokura Nature.395.(1998).677.

5) C.L. Yuan, S.G. Wang, W.H. Song, T. Yu, J.M. Dai, S.L. Ye, Y.P. Sun, Appl Phys Lett 75.(1999).3853.

6) K.I. Kobayashi, .T Kimura, H. Sawada, K. Terakura and Y. Tokura. Phys Rev B.59.(1999).11159.

7) T.H. Kim, M. Uehara, S.W. Cheong and S. Lee. Appl Phys Lett .74.(1999).1737.

8) Y. Tomioka, T. Okuda, Y. Okimoto, R. Kumai and K.I. Kobayashi. Phys Rev B. 61.(2000).422.

9) D. Niebieskikwiat, A. Caneiro, R.D. Sanchez and J. Fontcuberta. Phys Rev B. 64.(2001). 180406.

10) A. Sharma, A. Berenov,J. Rager, W. Branford, Y. Bugosiavsky, L.F. Cohen, and J.L. MacManus-Driscoll. Appl Phys Lett .83.(2003).2384.

11) K.Wang and Y. Sui. Solid State Commun 129.(2004).135.

12) C.L. Yuan, Y. Zhu, P.P. Ong, Z.X. Shen and C.K. Ong. Solid State ommun.129.(2004).551.

13) C.L. Yuan, Y. Zhu and P.P. Ong. Appl Phys Lett .82.(2003).934.

14) M.X. Dai, C. Su, R. Wang and Z.L. Wang. Solid State Communications. 119.(2001).377.

15) J. Raittila, T. Salminem, $T$. Suominem, K. Schlesier and P. Paturi. J Phys Chem Solids. 67.(2006).1712.
16) D. Demirskyi, H Borodianska,D.; Agrawal, A. Ragulya, Y.Sakka and O. Vasylkiv, J. Alloys Comp. 523 (5). (2012).1.

17) A. Agostino, E. Bonometti, P. Volpe, M. Truccato, C. Manfredotti, P. Olivero, C.Paolini, G. Rinaudo and L. Gozzelino. Int. J. Mod. Phys. B. 17 (4-6). (2003).773.

18) C. Dong, J.Guo, G. C.Fu, L. H.Yang and H. Chen. Supercond. Sci. Technol. 17 (12). (2004). L55.

19) Q.Xia, J.Yi, Y.Peng, S.Luo and L. Li. Mater. Lett. 62 (24).(2008). 4006.

20) J. H.; Lim, S. H.; Jang, S. M.; Hwang, J. H.; Choi, J.; Joo, W. N.; Kang and C. Kim. Physica C .468.(2008). 1829.

21) T. Nakagawa, K. Yoshikawa and S. Nomura.J Phys Soc Jpn. 27. (1969).880. Pattern No 04-002-3154. Calculated from LPF using POWD$12++$.

22) H. Sakuma, T. Taniyama, Y. Kitamoto and Y. Yamazaki. J Appl Phys. 93.(2003).2816.

23) C.Plapcianu, A. Agostino,P. Badica,G. V. Aldica,E. Bonometti,G. Ieluzzi,S. Popa, M. Truccato, S. Cagliero, Y. Sakka,O. Vasylkiv and R. Vidu, Ind.Eng.and Chem. Res.51. (2012). 11005

24) M.Cernea, F. Vasiliu, C. Plapcianu, C. Bartha, I. Mercioniu, I. Pasuk, R.Lowndes, R. Trusca, G. V. Aldica and L. Pintilie. J.of European Ceramic Society. 33. (2013). 2483

25) H.Y. Hwang, S.W. Cheong, N.P. Ong and B. Batlogg. Phys Rev Lett. 77.(1996).2041. 